\title{
SNP- and Haplotype Analysis of the Tryptophan Hydroxylase 2 Gene in Alcohol-Dependent Patients and Alcohol-Related Suicide
}

\author{
Peter Zill',', Ulrich W Preuss',2, Gabrielle Koller', Brigitta Bondy' and Michael Soyka' \\ 'Department of Psychiatry, Ludwig-Maximilians-University, Munich, Germany; ${ }^{2}$ Department of Psychiatry, Martin-Luther-University, Halle, \\ Halle/Saale, Germany
}

\begin{abstract}
Several lines of evidence indicate that disturbances of the central serotonergic system are involved in the pathophysiology of alcohol dependence and suicidal behavior. Recent studies have indicated that a newly identified second isoform of the tryptophan hydroxylase gene (TPH2) is preferentially involved in the rate limiting synthesis of neuronal serotonin. Genetic variations in the TPH2 gene have been associated with an increased risk for major depression and suicidal behavior. We performed single SNP (single nucleotide polymorphism), linkage disequilibrium and haplotype studies on 353 alcohol-dependent patients of whom I02 individuals had a history of at least one suicide attempt and 305 healthy controls with 20 SNPs covering the entire gene region of TPH2. Neither single SNP-, nor haplotype analysis could detect significant associations with alcohol dependence and/or suicidal behavior among alcohol-dependent patients. One major haplotype block of strong linkage disequilibrium between introns 5 and 8 of the TPH 2 gene has been found in alcoholics and controls, which is in concordance with recent reports. In conclusion, our results suggest that single SNPs, respectively, haplotypes of the TPH2 gene are unlikely to play a major role in the pathophysiology of alcohol dependence or the alcoholism-related phenotype suicidal behavior. Further analysis are needed to confirm these results.

Neuropsychopharmacology (2007) 32, 1687- 1694; doi: I0.1038/sj.npp. I 30 I 3 I ; published online 24 January 2007
\end{abstract}

Keywords: tryptophan hydroxylase; alcoholism; suicide; haplotypes; association study; polymorphism

\section{INTRODUCTION}

There is growing evidence that alcohol dependence is a multifactorial disorder probably caused by genetic and environmental factors, as well as their interactions (Dick and Foroud, 2003). Despite a strong evidence for genetic effects, specific genes that modulate the risk for alcoholism, as well as for alcoholism-related phenotypes have still not been identified.

There is no doubt that the serotonergic system is involved in the regulation of alcohol preference and intake in humans and animals (Naranjo et al, 1986). Several human studies have implicated alterations in the serotonergic neurotransmission in the etiology of alcoholism (Murphy, 1990; Virkkunen and Linnoila, 1997), for instance lower levels of 5-hydroxy indole acetic acid (5-HIAA) in the cerebrospinal fluid of alcoholics have been reported (Banki,

\footnotetext{
*Correspondence: Dr P Zill, Department of Psychiatry, Psychiatric Hospital of the Ludwig-Maximilians-University, Nussbaumstr. 7, D-80336 Munich, Germany, Tel: + 4989 5160274l, Fax: + 4989 5160474I, E-mail: Peter.Zill@med.uni-muenchen.de

Received II April 2006; revised 19 October 2006; accepted 27 November 2006
}

1981). Furthermore, pharmacological agents that increase serotonin (5-HT) also cause a reduction in alcohol self administration both in rats and humans (LeMarquand et al, 1994).

Additionally, numerous genetic studies of serotonergic candidate genes for alcohol dependence and/or alcoholrelated phenotypes have been published, investigating mainly genes encoding the serotonin transporter $(5-\mathrm{HTT})$ or serotonin receptors. While the role of the 5-HTT gene in the pathophysiology of alcohol dependence remains controversial, no evidence exists for an association between alcoholism- or alcoholism-related phenotypes with any of the 5-HT receptor genes (Dick and Foroud, 2003).

In this context, a further interesting candidate gene is the recently new identified neuronal tryptophan hydroxylase 2 gene (TPH2), the rate limiting enzyme in the serotonin synthesis (Walther et al, 2003). In a post-mortem study, we were recently able to demonstrate that TPH2 mRNA is also expressed in several regions of the human brain, as frontal cortex, thalamus, hippocampus, hypothalamus, amygdala, cerebellum and raphe nuclei but not in peripheral tissues such as heart, lung, kidney, duodenum, liver and adrenal gland in contrast to TPH1 (Zill et al, 2004b). A comparative analysis of TPH1 and TPH2 demonstrated that the mRNA of 
both genes is expressed in each investigated brain region with variations between the brain areas, as well as between the particular genes. The major finding of this study was the detection of the highest TPH2 mRNA levels in the raphe nuclei ( $\sim 4$-fold more abundant than that of TPH1) (Zill et al, 2007). These findings might implicate a duality of the serotonergic system with peripheral serotonergic effects determined by TPH1 and the CNS effects regulated by TPH 2 including a partially uncoupling of behavioral effects from TPH1.

Additional support for an involvement of the TPH2 gene in behavioral traits can be derived from genetic studies. In a SNP- and haplotype association study between the TPH2 gene and major depression we have genotyped 10 SNPs between exon 5 and exon 7 in 300 depressed patients and 265 healthy controls and found significant association in both single SNP and haplotype analysis (Zill et al, 2004a). This finding could later on be replicated by Harvey et al (2004) in a haplotype analysis with SNPs, overlapping the region of our study. Interestingly, a further association analysis of our own group using the same SNPs as described above with 263 individuals who committed suicide and 265 healthy controls provided also evidence for association with suicidal behavior (Zill et al, 2004c).

Completed suicide, as well as suicide attempts are reported to be significantly increased in alcohol dependence (Preuss et al, 2002). One of the most important risk factors for suicidal behavior is a comorbid diagnosis of an alcohol use disorder, which increases the risk for completed suicide up to 60- to 120-fold (Murphy and Wetzel, 1990).

With regard to the repeatedly described comorbidity of suicidality-alcoholism and the abovementioned genetic findings in the serotonin-related disorders depression and suicidal behavior, the TPH2 gene represents not only an interesting candidate gene for alcohol dependence, but also for suicidal behavior among alcohol-dependent patients.

Therefore, the aim of the present study was to investigate a probable role of polymorphisms in the TPH2 gene in the etiology of alcohol dependence, as well as in suicide attempts in alcohol-dependent patients. We performed association and linkage disequilibrium studies on 353 alcohol-dependent patients including 102 individuals with at least one suicide attempt and 305 healthy controls with 20 SNPs covering the entire gene region of TPH2. To enhance the detection power of association, we compared also estimated haplotype frequencies deduced from genotype frequencies of the 20 SNPs.

To our knowledge this is the first study with the TPH2 gene in alcoholism and alcoholism-related phenotypes.

\section{MATERIALS AND METHODS}

\section{Subjects}

A total of 353 alcohol-dependent patients (274 male patients, 79 female patients) were recruited from the Addiction Treatment Ward of the Psychiatric Department of the Ludwig-Maximilians-University Munich. All patients had been admitted for the treatment of alcohol dependence, were unrelated, of German descent and older than 18 years. The patients met both, the ICD-10 and DSM-IV criteria of alcohol dependence assessed with a structured interview (SCID-II: structured clinical interview according to DSM IV, German version) (Wittchen et al, 1997). The criteria of alcohol dependence, history of suicide attempts and depression were assessed using the SSAGA (Semi-Structured Interview for Assessment of Genetics in Alcoholism) (Bucholz et al, 1994; Hesselbrock et al, 1999) and by a comprehensive psychiatric examination.

The SSAGA provides items regarding the age of onset of first suicide attempts, the number of attempts and the method used. The attempts were classified according to violence of the method. Overdose due to alcohol or sedative intake was considered nonviolent; shooting, immolation drowning, cutting, jumping and hanging were considered violent suicide attempts. Further data, as history of major or minor depression, depressive episodes and first age of onset were also obtained using the SSAGA. Interrater reliability was not assessed in this study, since several manuscripts reported sufficient interrater reliability for the SCID II interview for several international versions of the SCID II (Zanarini et al, 2000; Maffei et al, 1997), including the translated and validated German Version (Mestel et al, 2001). As the full interview was conducted by experienced psychiatrists, the validity of the personality diagnoses should be even higher than by using the SCID II self-rating questionnaire alone.

For the SSAGA, an evaluation of the German Version was published several years ago (Keppel et al, 2001) and reported interrater reliabilities for alcohol and substance dependence of 0.85 (DSM-III-R) and 0.95 (ICD-10). For the American version which originates from 'The COGA-Study: Collaborative Study on Genetics in Alcoholism' (Edenberg and Foroud, 2006), two previous articles were published investigating psychometric properties of this semi-structured interview (Bucholz et al, 1994; Hesselbrock et al, 1999).

All patients were examined two weeks after admission, were free of any withdrawal symptoms and psychopharmacological treatment. Patients with current polysubstance abuse were excluded from the study. Past and present polysubstance abuse were assessed using the specific SSAGA module. The exclusion of all alcoholic patients with other concurrent axis-I disorders, as anxiety or depressive symptoms would decrease the power of the study significantly and limit its validity, since anxious and depressive symptoms occur in high rates among inpatient alcoholdependent patients. As the methodology of this study was compatible to the COGA-project, only subjects with current or previous bipolar, schizoaffective or schizophrenic psychosis were excluded. These disorders are significantly less frequent in alcoholics.

In all, 305 ethnically matched subjects of Caucasian origin from the general population in southern Germany (164 male patients, 141 female patients) served as control group. Control persons were recruited from the general population at different locations (eg, libraries, road construction sites, department stores) and represent a range of social classes from unskilled workers to university graduates.

A comprehensive medical and psychiatric assessment including the SSAGA, as well as personality questionnaires (MMPI, NEO-FFI, TCI) and a short structured clinical interview with a psychiatrist was carried out in all control 
Table I Demographic Characteristics of the Alcohol-Dependent Patients (ALC) and Healthy Controls (CON)

\begin{tabular}{|c|c|c|}
\hline & ALC $(N=353)$ & $\operatorname{CON}(N=305)$ \\
\hline Sex (males/females) & $274 / 79$ & $|64 /| 4 \mid$ \\
\hline Age (years) ${ }^{\mathrm{a}}$ & $42.7 \pm 9.7$ & $40.05 \pm 15.2$ \\
\hline Age of onset (years) ${ }^{a}$ & $30.4 \pm 9.4$ & \\
\hline Suicide attempts & 102 & \\
\hline Violent/non violent & $58 / 44$ & \\
\hline Daily alcohol intake (g/day) $)^{a}$ & $310.8 \pm 182.3$ & \\
\hline
\end{tabular}

${ }^{\mathrm{a}}$ Data are mean values $\pm \mathrm{SD}$.

persons together with routine laboratory screening to assess general dimensional personality characteristics and to exclude possible psychiatric axis I/II disorders, such as schizophrenia, depression, personality disorders and substance use disorders including alcohol dependence. Also persons with severe physical diseases were not included in the study. This control sample has already been used in further studies (Zill et al, 2004a, c).

All persons with any first-degree relative with a history of any axis I disorders (including alcohol dependence) were also excluded. Histories of psychiatric disorders in firstdegree relatives of patients and controls were assessed employing the FHAM (Family History Assessment Module) (Rice et al, 1995), an instrument from the COGA-Study which was translated and back-translated by our research group. Demographic data of the alcohol-dependent patients and the control group are given in Table 1 .

The local ethical committee of the Ludwigs-MaximiliansUniversity of Munich approved the study protocol; patients and controls were included in the study after they gave written informed consent.

\section{Genotyping}

Genomic DNA was isolated from whole blood according to standard procedures. The human TPH2 gene is located on chromosome 12q15, comprises 11 exons and covers a region of $\sim 93 \mathrm{kbp}$. As the TPH2 gene is highly homologous to the TPH1 gene, especially in the coding regions (exons), we have only chosen intronic SNPs to avoid co-amplification of TPH1 gene sequences. 20 intronic SNPs, which cover the entire gene region were chosen from the public data base (http://www.ncbi.nlm.nih.gov/SNP/). Additionally the TPH2 specificity of the primers were tested by a BLAST search applying the ENSEMBL BLAST VIEW program (http://www.ensembl.org/Multi/blastview). Table 2 summarizes the investigated SNPs, their localization, distance and nucleotide exchange. We analyzed the same SNPs as described previously (Zill et al, 2004c) including 10 further SNPs to cover the entire gene region. SNPs were chosen according to reported allele frequencies in the database with an average distance between 5 and $10 \mathrm{kpbs}$ to cover the relevant bins.

The 20 SNPs in the TPH2 gene were genotyped using the snapshot methodology (Applied Biosystems, Foster City, CA, USA). This is a commercially available mini sequencing method that relies upon the extension of a primer
Table 2 Investigated SNPs and their Localization in the THP2 Gene

\begin{tabular}{|c|c|c|c|c|}
\hline Marker & SNP ID ${ }^{a}$ & $\begin{array}{l}\text { Position relative to } \\
\text { transcription start } \\
\text { site of } \mathrm{TPH}^{\mathrm{b}} \text { (bp) }\end{array}$ & $\begin{array}{c}\text { Gene } \\
\text { location }\end{array}$ & Polymorphism \\
\hline A & rs4570625 & -703 & $5^{\prime}-$ UTR & $\mathrm{G} / \mathrm{T}$ \\
\hline B & rs434I58I & 2448 & intron I & $\mathrm{G} / \mathrm{T}$ \\
\hline C & rs2129575 & 7448 & intron 4 & $\mathrm{G} / \mathrm{T}$ \\
\hline D & rs|386488 & 11993 & intron 5 & $\mathrm{~A} / \mathrm{C}$ \\
\hline E & rs|843809 & 16073 & intron 5 & $\mathrm{G} / \mathrm{T}$ \\
\hline$F$ & rs 2220330 & 19083 & intron 5 & $\mathrm{G} / \mathrm{A}$ \\
\hline G & rs|386495 & 19697 & intron 5 & $\mathrm{C} / \mathrm{T}$ \\
\hline $\mathrm{H}$ & rs|386494 & 19918 & intron 5 & $A / G$ \\
\hline । & rs6582072 & 21852 & intron 5 & $A / G$ \\
\hline J & rs|386493 & 22554 & intron 5 & $\mathrm{C} / \mathrm{T}$ \\
\hline K & rs|386492 & 29640 & intron 5 & $A / G$ \\
\hline L & rs $47608 \mid 4$ & 34309 & intron 6 & $A / G$ \\
\hline M & rs47608I5 & 39604 & intron 6 & T/A \\
\hline$N$ & rs 4760750 & 45265 & intron 7 & $\mathrm{~A} / \mathrm{C}$ \\
\hline O & rs 10506645 & 52875 & intron 7 & $\mathrm{C} / \mathrm{T}$ \\
\hline$P$ & rs I 386497 & 59665 & intron 8 & $\mathrm{~A} / \mathrm{C}$ \\
\hline Q & rs| 487278 & 68226 & intron 8 & $\mathrm{C} / \mathrm{T}$ \\
\hline$R$ & rs9325202 & 74852 & intron 8 & $A / G$ \\
\hline S & rs|386486 & 79595 & intron 8 & $\mathrm{C} / \mathrm{T}$ \\
\hline T & rs| 487280 & 86202 & intron 9 & $A / G$ \\
\hline
\end{tabular}

${ }^{a} \mathrm{SNP}$ ID number from the SNP database (http://www.ncbi.nlm.nih.gov/SNP/).

bFrom the Genbank contig NT_029419 (orientation: genomic forward)

(http://www.ncbi.nlm.nih.gov/).

immediately adjacent to the SNP using fluorescently labeled dideoxy nucleotides. The fluorescently labeled extension primers can then be visualized by electrophoresis on an capillary PRISM 310 automated sequencer (Applied Biosystems). Turner et al (Turner et al, 2002) provides a good overview about this method. Detailed information on PCR primers and extension primer sequences as well as reaction PCR conditions can be obtained on request. Data were processed by using Genscan Analysis version 3.7 and Genotyper version 3.7 (Applied Biosystems).

All laboratory procedures were carried out blind to case control status.

\section{Statistics}

All genotyping results were tested for Hardy-Weinberg Equilibrium (HWE) applying the HWSIM computer program (http://krunch.med.yale.edu/hwsim).

Single SNP association analysis and calculation of pair wise linkage disequilibrium (LD) between the SNPs was performed with the computer program COCAPHASE 2.35 (http://www.hgmp.mrc.ac.uk) (Dudbridge, 2003). We used $\mathrm{D}^{\prime}$ to describe the magnitude of LD (Lewontin, 1988). This package calculates likelihood ratio tests under a log-linear model of the probability that an allele belongs to the case rather than the control group, using a standard unconditional logistic regression. The global null hypothesis that all odds ratios are equal was tested by permutation. This 
method randomly reassigns the case and control labels in the actual data and gives a significance level corrected for all markers tested. Permutations (10000) were performed in each permutation analysis.

Estimation of haplotype frequencies was done with the computer program PHASE 2.1 (http://www.stat.washington.edu/stephens/software.html) (Stephens et al, 2001). This program implements a Bayesian statistical method for reconstructing haplotypes from genotype data and has the advantage that the mean error rate is approximately half than obtained with the expectation maximization (EM) algorithm method. Statistical comparison of the haplotype frequencies was performed by $\chi^{2}$-test applying the CLUMP computer program (http://linkage.rockefeller.edu/soft/ clump.html). CLUMP is a program designed to assess the significance of the departure of observed values in a contingency table from the expected values conditional on the marginal totals. The present implementation works on $2 \times \mathrm{N}$ tables and was designed for use in genetic casecontrol association studies (Sham and Curtis, 1995). In the case of a significant global test, individual haplotypes were tested for association by grouping all others together and applying a $\chi^{2}$-test with $1 \mathrm{df}$.

The significance level for all statistical tests was 0.05 . We applied Bonferroni corrections for all multiple tests.

Power was estimated by the computer program GENETIC POWER CALCULATOR (http://statgen.iop.kcl.ac.uk/cgi-bin/ powercalc/cc2.cgi) (Purcell et al, 2003).

\section{Nucleotide Sequences}

The SNP are named in the present study according to the ID numbers from the SNP database (http://www.ncbi. nlm.nih.gov/SNP/). Position of the SNPs were taken from the Genbank contig: accession number NT_029419 (http:// www.ncbi.nlm.nih.gov/) (Table 2).

\section{RESULTS}

We performed an association study with 20 SNPs covering $\sim 87 \mathrm{kbp}$ of the entire gene region of TPH2 $(93 \mathrm{kbp})$ in a sample of 353 alcohol-dependent patients which was composed of 102 patients with and 251 patients without a history of at least one suicide attempt and 305 healthy controls. We compared the total group of alcohol-dependent patients $v s$ the controls, the suicide subgroups $v s$ the control group and the alcohol-dependent patients with $v s$ without a suicide attempt.

All SNPs were found to be in Hardy-Weinberg equilibrium in the cases and control samples.

\section{Single Marker Association Analysis}

The allele frequency distribution for each SNP is presented in Table 3. We could not detect any significant differences in the allele frequencies between the four groups either by single $\chi^{2}$-statistics, nor by global permutation tests. Similar results were also obtained for the comparison of the genotypes (data not shown). Power analysis showed that for the detection of a main effect for one of the 20 polymorphisms with a relative risk of 1.5, given the disorder related gene frequency of 0.15 and a test size of $\alpha=0.05$, our sample of alcohol-dependent patients has a power of $90 \%$. For the comparative analysis between the two subgroups of alcohol-dependent individuals with and without a history of suicide attempts considering the above-described conditions, we obtained a power of $80 \%$. Therefore we had sufficient power to detect such an effect.

\section{Inter Marker Linkage Disequilibrium}

The standardized measure of linkage disequilibrium (LD) denoted as $\mathrm{D}^{\prime}$ were calculated for all pairs of SNPs on both the total sample of alcohol-dependent patients and the controls. Tables 4 and 5 present these data. The LD pattern is fairly identical in both samples, and nearly completely homogeneous. Most of the SNPs were in tight and partially complete LD with each other. The LD analysis yielded one major block of strong linkage disequilibrium between SNP $\mathrm{D}$ (rs1386488) in intron 5 and SNP Q (rs1487278) in intron 8 in both samples.

\section{Haplotype Analysis}

Due to the strong LD between SNP D (rs1386488) and SNP $\mathrm{Q}$ (rs1487278) a 14-marker haplotype analysis was performed with all SNPs of this LD block across the TPH2 gene. Table 6 demonstrates the frequencies for the estimated 14-marker-haplotypes among controls, alcohol-dependent patients and the subgroups with and without a suicide attempt. Only haplotypes which occur with a frequency $\geqslant 0.01$ in one of the four groups were considered. We observed three major haplotypes which account for $86 \%$ of all possible marker combinations in the total patient sample, respectively, $90 \%$ in the control sample. Comparable to the single SNP evaluation we could not detect any significant differences in the haplotype distribution comparing the four samples.

\section{DISCUSSION}

We performed an association study, applying SNP-, LDand haplotype analysis of the newly identified second tryptophan hydroxylase isoform (TPH2) gene in 353 patients suffering from alcohol dependence and 305 healthy controls. Owing to our previous findings about a significant single SNP- and haplotype association between polymorphisms in the TPH2 gene and completed suicide (Zill et al, 2004c) we included also alcohol-dependent patients with a history of at least one suicide attempt in the present study and analyzed them separately $v s$ healthy controls and versus alcohol-dependent patients without a suicide attempt.

Single SNP analysis revealed no significant relationship, but given that the investigated SNPs are common in the general population and with regard to the heterogeneity of these disorders, it is probable that single SNPs do not represent the primary basis of the disease, but rather SNP combinations (haplotypes) should be considered. Using 14 SNPs within a strong haplotype block between the SNPs D (rs1386488) in intron 5 and Q (rs1487278) in intron 8 across the TPH2 gene we observed three major haplotypes, which account for $86 \%$ of all possible marker combinations in the total patient sample, respectively, $90 \%$ in the control 
Table 3 Allele Frequencies among Controls (CON), all Alcohol-Dependent Patients (ALC total), Patients with and without a History of at least One Suicide Attempt

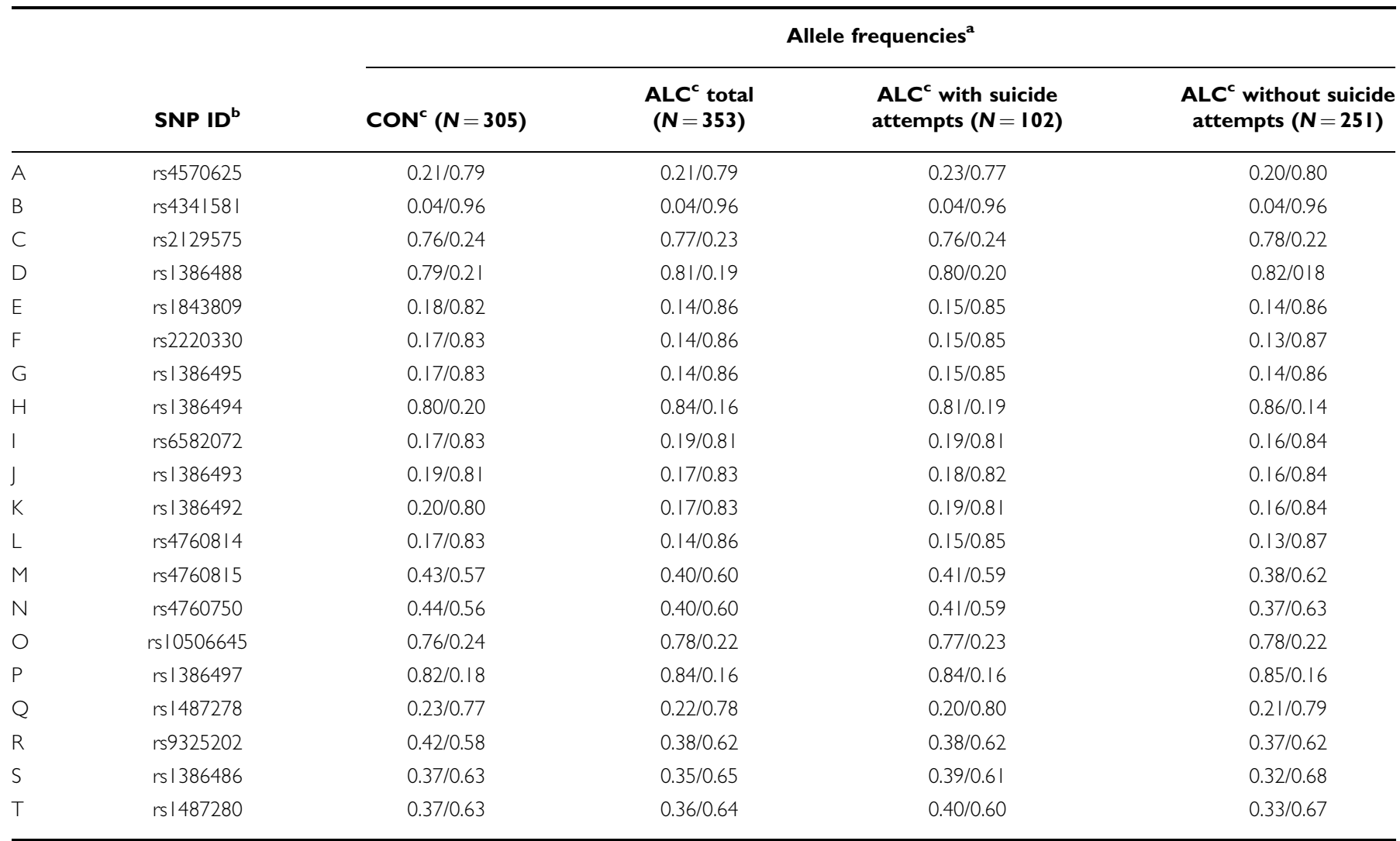

aShown for both alleles in the order allele I/allele 2 as listed in Table 2

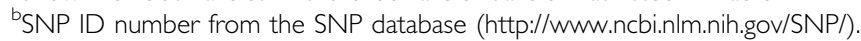

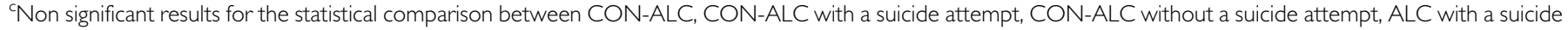
attempt-ALC without a suicide attempt.

Table 4 Estimated Haplotype Frequencies of the I4 SNP Haplotype of SNP D-Q among Controls (CON), all Alcohol-Dependent Patients (ALC Total), Patients with and without a History of at least One Suicide Attempt (Only Haplotypes which Occur with a Frequency $\geqslant 0.01$ in One of the Four Groups were Considered)

Estimated haplotype frequencies

\begin{tabular}{ccccl}
\hline $\begin{array}{c}\text { CON } \\
(\mathbf{N}=\mathbf{3 0 5})\end{array}$ & $\begin{array}{c}\text { ALC total } \\
(\mathbf{N}=\mathbf{3 5 3})\end{array}$ & $\begin{array}{c}\text { ALC with } \\
\text { suicide } \\
\text { attempts } \\
(\mathbf{N}=\mathbf{~} \mathbf{0 2})\end{array}$ & $\begin{array}{c}\text { ALC without } \\
\text { suicide } \\
\text { attempts } \\
(\mathbf{N}=\mathbf{2 5} \mathbf{l})\end{array}$ & Global $\boldsymbol{P}$-value \\
\hline 0.49 & 0.56 & 0.53 & 0.56 & CON vs ALC with suicide attempts: ns \\
0.21 & 0.21 & 0.19 & 0.21 & \\
0.16 & 0.13 & 0.15 & 0.13 & CON vs ALC total: ns \\
0.02 & 0.03 & 0.03 & 0.02 & CON vs ALC without suicide attempts: ns \\
0.01 & 0.02 & 0 & 0.02 & ALC with suicide attempts vs \\
0.02 & 0 & 0.03 & 0 & ALC with-out suicide attempts: ns \\
\hline
\end{tabular}

Based on the physical marker order: from rs|386488 (SNP D, intron 5) to rs| 487278 (SNP Q, intron 8).

sample. Haplotype analysis yielded no significant differences within a comparison of the four investigated samples.

The power of haplotype analysis is dependent on LD between relevant markers. To estimate LD between pairs of SNPs we used Lewontin's D' (Lewontin, 1988). D' has been shown to be reasonable robust towards differences in allele frequencies in contrast to $\mathrm{D}$ (Thompson et al, 1988). The LD pattern of all the SNP combinations is fairly identical in both samples and shows the often observed block pattern in the genome, which can be explained by the existence of a 
Table 5 D'-Values for all Combinations of the TPH2 SNPs in Alcohol-Dependent Patients

\begin{tabular}{|c|c|c|c|c|c|c|c|c|c|c|c|c|c|c|c|c|c|c|c|c|}
\hline $\mathrm{SNPs}^{\mathrm{a}}$ & A & B & C & D & $E$ & $\mathbf{F}$ & $\mathbf{G}$ & $\mathbf{H}$ & I & J & $K$ & $\mathbf{L}$ & $M$ & $\mathbf{N}$ & 0 & $\mathbf{P}$ & $\mathbf{Q}$ & $\mathbf{R}$ & $\mathbf{S}$ & $\mathbf{T}$ \\
\hline A & & 1.00 & 0.91 & 0.01 & 0.83 & 0.82 & 0.83 & 0.57 & 0.02 & 0.03 & 0.04 & 0.81 & 0.91 & 0.90 & 0.77 & 0.87 & 0.69 & 0.62 & 0.47 & 0.47 \\
\hline B & & & 1.00 & 1.00 & 0.73 & 0.73 & 0.73 & 0.77 & 0.76 & 0.76 & 0.76 & 0.73 & 0.68 & 0.61 & 0.81 & 0.72 & 0.46 & 0.56 & 0.62 & 0.61 \\
\hline C & & & & 0.21 & 1.00 & 1.00 & 1.00 & 0.69 & 0.08 & 0.06 & 0.03 & 1.00 & 0.84 & 0.83 & 0.78 & 0.57 & 0.74 & 0.70 & 0.48 & 0.48 \\
\hline D & & & & & 1.00 & 1.00 & 0.99 & 0.89 & 1.00 & 1.00 & 0.98 & 1.00 & 0.88 & 0.85 & 1.00 & 0.86 & 1.00 & 0.67 & 0.63 & 0.64 \\
\hline$E$ & & & & & & 1.00 & 0.99 & 0.99 & 0.95 & 0.96 & 0.97 & 0.99 & 0.98 & 0.98 & 1.00 & 0.98 & 1.00 & 0.96 & 0.74 & 0.76 \\
\hline $\mathrm{H}$ & & & & & & & & & 0.85 & 0.86 & 0.87 & 0.99 & 0.83 & 0.81 & 0.84 & 0.88 & 0.81 & 0.81 & 0.62 & 0.64 \\
\hline । & & & & & & & & & & 0.99 & 1.00 & 0.97 & 1.00 & 0.98 & 1.00 & 0.84 & 1.00 & 0.79 & 0.77 & 0.78 \\
\hline J & & & & & & & & & & & 1.00 & 0.99 & 1.00 & 0.98 & 1.00 & 0.85 & 1.00 & 0.79 & 0.76 & 0.77 \\
\hline K & & & & & & & & & & & & 1.00 & 1.00 & 0.98 & 1.00 & 0.86 & 1.00 & 0.76 & 0.76 & 0.77 \\
\hline$P$ & & & & & & & & & & & & & & & & & 1.00 & 0.98 & 0.63 & 0.65 \\
\hline Q & & & & & & & & & & & & & & & & & & 1.00 & 0.52 & 0.54 \\
\hline $\mathrm{R}$ & & & & & & & & & & & & & & & & & & & 0.62 & 0.63 \\
\hline S & & & & & & & & & & & & & & & & & & & & 0.99 \\
\hline
\end{tabular}

aBased on the physical marker order: from rs4570625 (A) to rs I487280 (T) dark gray: $D^{\prime} \geqslant 0.90$; light gray: $D^{\prime} \geqslant 0.80 ;$ white: $D^{\prime}<0.80$.

Table 6 D'-Values for all Combinations of the TPH2 SNPs in Controls

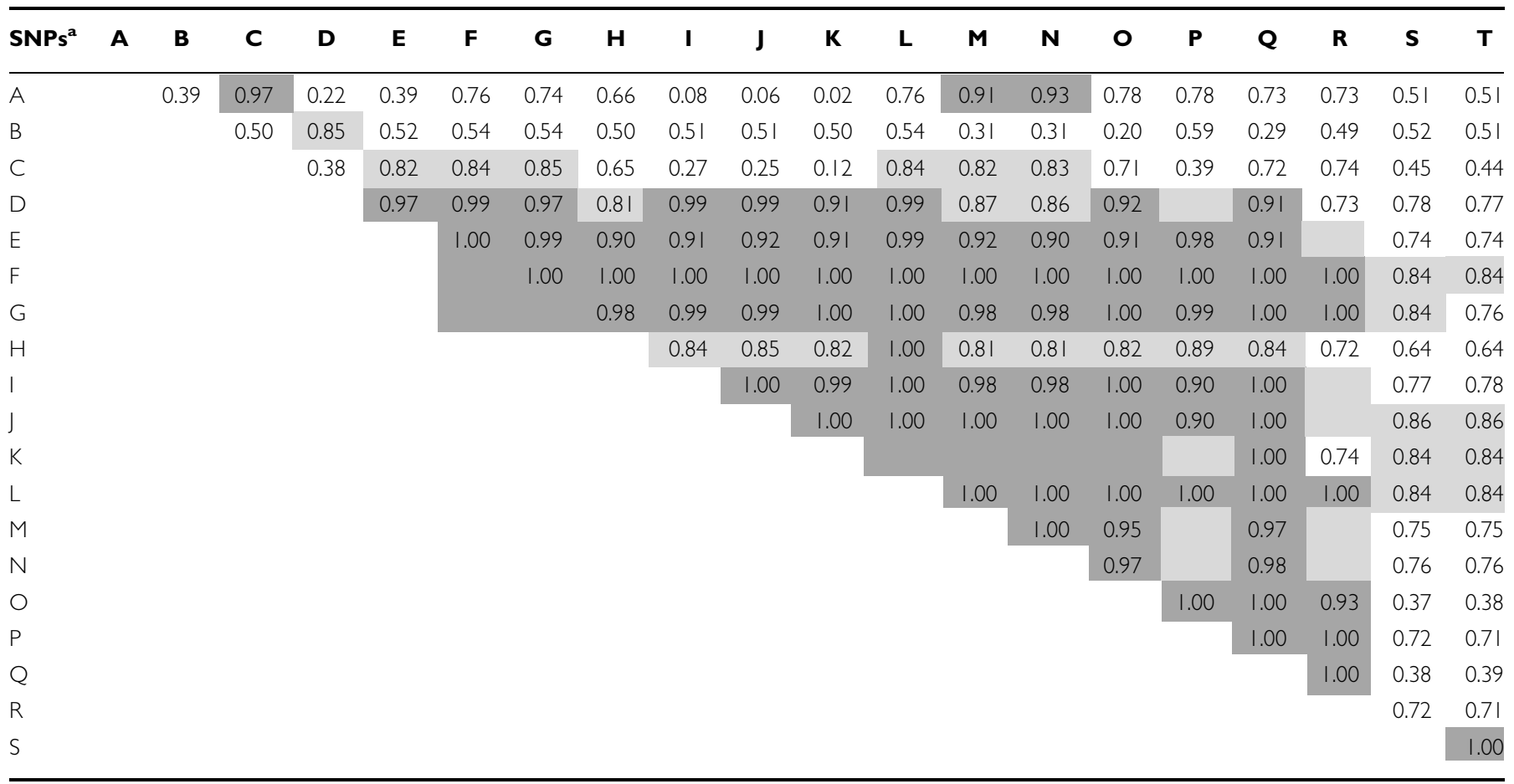

${ }^{a}$ Based on the physical marker order: from rs4570625 (A) to rs 1487280 (T) dark gray: $\mathrm{D}^{\prime} \geqslant 0.90$; light gray: $\mathrm{D}^{\prime} \geqslant 0.80 ;$ white: $\mathrm{D}^{\prime}<0.80$.

few common haplotypes that account for most of the haplotype diversity (Gabriel et al, 2002). The LD analysis yielded one major block of strong linkage disequilibrium between SNP D (rs1386488; intron 5) and SNP Q (rs1487278, intron 8) in the control sample, as well as in the total group of alcohol-dependent patients. Simulation studies have estimated the length of useful LD to be as low as $3 \mathrm{~kb}$ (Kruglyak, 1999), whereas LD more than $60 \mathrm{~kb}$ from 
common alleles was observed in 19 randomly selected genomic regions (Reich et al, 2001). Therefore, we should have sufficient power in our haplotypes study. Moreover, our results are in concordance with previous findings by Zhou et al (2005), who found comparable haplotype blocks between intron 5 and intron 8 in US and Finish whites, in African Americans and in southwestern American Indians and are consistent with our recent results in German depressive patients, suicide victims and healthy controls (Zill et al, 2004a, c).

Interestingly, we could not find any relation between SNPs or haplotypes of the TPH2 gene and the existence of suicidal behavior in alcohol-dependent patients. This hypothesis is inconsistent with our previous results about an association between specific SNPs and haplotypes of the TPH2 gene and completed suicide (Zill et al, 2004c). This is not surprising if one considers that the definition of suicidal behavior is not as clear cut as desired for genetic studies. This may lead to spurious findings due to insufficient or illdefined phenotypes. It has been reported that a serotonergic dysfunction might have an increased magnitude in subjects who have attempted or completed suicide using violent means (Courtet et al, 2005). Therefore, under the assumption that suicidal behavior is a complex disorder and susceptibility is determined by the action of several genes that interact with environmental factors it is likely that suicide attempts and completed suicide are rather different phenotypes concerning their genetic factors. Moreover, the phenotype definition of the present study 'at least one suicide attempt' might be too heterogeneous. In our sample of 102 alcohol-dependent patients with a suicide attempt, $43 \%$ tried to commit suicide in a nonviolent manner and $57 \%$ in a violent manner. Owing to the limited number of patients in these two subgroups, we were unfortunately not able to distinguish between the methods in the genetic analysis.

On the other hand also the diagnosis 'alcoholism' might be too unspecific for genetic studies. A determination of the type of alcohol dependence according to Babor in type A and B would be probably more indicated (Babor et al, 1992). The Babor-classification has significant overlaps with the Cloninger-typology (Cloninger et al, 1988) and seems to be more suitable for clinical studies. This could already be demonstrated with the present sample (Bottlender et al, 2006). Unfortunately, the present sample size is too small for a first classification according to the alcohol-dependence type and a further sub grouping into suicidal behavior groups. The resulting groups are not adequate for genetic studies.

To our knowledge up to now only the study by Zhou et al (2005) has investigated a probable association between alcoholism and TPH2 gene variants. Similar to our present results this group could not demonstrate a relation between single SNPs and haplotypes of the TPH2 gene and alcohol dependence neither in Finish nor in African-American populations.

A possible limitation of the present study is the fact that all case-control association studies have a risk of false positive or negative findings due to population stratification (Schulze and McMahon, 2002), although the extent to which such stratification actually contributes to false negative findings is controversial (Cardon and Palmer, 2003).
Further studies with so-called 'parent-child-trios' are needed to circumvent these problems and to confirm our results.

In summary, our results suggest that single SNPs, respectively haplotypes of the TPH2 gene are unlikely to play a major role in the pathophysiology of alcohol dependence or the alcoholism-related phenotype suicidal behavior. Numerous studies with polymorphisms in the TPH1 gene in alcohol dependence have tried to link genetic variants to this disease, but the results remain inconclusive (Parsian and Cloninger, 2001; Fehr et al, 2001; Koller et al, 2005; Nielsen et al, 1998; Anghelescu et al, 2005). Therefore, possible interactions between TPH1 and TPH2 should also be considered as modulating mechanisms in the pathogenesis of alcohol-dependence and alcohol-related phenotypes. Moreover the involvement of recently published functional polymorphisms (Brown et al, 2005; Zhang et al, 2005) in the TPH2 require further studies to confirm the present results.

\section{ACKNOWLEDGEMENTS}

We indicate that there is no possible financial or other conflict of interest related to this work. We thank Stefanie Behrens for her excellent assistance in laboratory work and data analysis.

\section{REFERENCES}

Anghelescu I, Klawe C, Fehr C, Singer P, Schleicher A, Himmerich $\mathrm{H}$ et al (2005). The TPH intron 7 A218C polymorphism and TCI dimension scores in alcohol-dependent patients: hints to nonspecific psychopathology. Addict Behav 30: 1135-1143.

Babor TF, Hofmann M, DelBoca FK, Hesselbrock V, Meyer RE, Dolinsky ZS et al (1992). Types of alcoholics, I. Evidence for an empirically derived typology based on indicators of vulnerability and severity. Arch Gen Psychiatry 49: 599-608.

Banki CM (1981). Factors influencing monoamine metabolites and tryptophan in patients with alcohol dependence. J Neural Transm 50: 89-101.

Bottlender M, Preuss UW, Soyka M (2006). Association of personality disorders with Type A and Type B alcoholics. Eur Arch Psychiatry Clin Neurosci 256: 55-61.

Brown SM, Peet E, Manuck SB, Williamson DE, Dahl RE, Ferrell RE et al (2005). A regulatory variant of the human tryptophan hydroxylase-2 gene biases amygdala reactivity. Mol Psychiatry 10: $884-888$.

Bucholz KK, Cadoret R, Cloninger CR, Dinwiddie SH, Hesselbrock VM, Nurnberger JI et al (1994). A new, semi-structured psychiatric interview for use in genetic linkage studies: a report on the reliability of the SSAGA. J Stud Alcohol 55: 149-158.

Cardon LR, Palmer LJ (2003). Population stratification and spurious allelic association. Lancet 361: 598-604.

Cloninger CR, Sigvardsson S, Gilligan SB, von Knorring AL, Reich T, Bohman M (1988). Genetic heterogeneity and the classification of alcoholism. Adv Alcohol Subst Abuse 7: 3-16.

Courtet P, Jollant F, Castelnau D, Buresi C, Malafosse A (2005). Suicidal behavior: relationship between phenotype and serotonergic genotype. Am J Med Genet C Semin Med Genet 133: 25-33.

Dick DM, Foroud T (2003). Candidate genes for alcohol dependence: a review of genetic evidence from human studies. Alcohol Clin Exp Res 27: 868-879.

Dudbridge F (2003). Pedigree disequilibrium tests for multilocus haplotypes. Genet Epidemiol 25: 115-121. 
Edenberg HJ, Foroud T (2006). The genetics of alcoholism: identifying specific genes through family studies. Addict Biol 11: 386-396.

Fehr C, Schleicher A, Szegedi A, Anghelescu I, Klawe C, Hiemke C et al (2001). Serotonergic polymorphisms in patients suffering from alcoholism, anxiety disorders and narcolepsy. Prog Neuropsychopharmacol Biol Psychiatry 25: 965-982.

Gabriel SB, Schaffner SF, Nguyen H, Moore JM, Roy J, Blumenstiel $\mathrm{B}$ et al (2002). The structure of haplotype blocks in the human genome. Science 296: 2225-2229.

Harvey M, Shink E, Tremblay M, Gagne B, Raymond C, Labbe M et al (2004). Support for the involvement of TPH2 gene in affective disorders. Mol Psychiatry 9: 980-981.

Hesselbrock M, Easton C, Bucholz KK, Schuckit M, Hesselbrock V (1999). A validity study of the SSAGA-a comparison with the SCAN. Addiction 94: 1361-1370.

Keppel S, Dilg C, Franke P (2001). Test-retest and interraterreliability of the semistructured interview of alcohol and drug addiction (SIGAD). Suchtmedizin 3: 217-223.

Koller G, Engel R, Preuss U, Karakesisoglou A, Zill P, Bondy B et al (2005). Tryptophan hydroxylase gene 1 polymorphisms are not associated with suicide attempts in alcohol-dependent individuals. Addict Biol 10: 269-273.

Kruglyak L (1999). Prospects for whole-genome linkage disequilibrium mapping of common disease genes. Nat Genet 22: 139-144.

LeMarquand D, Pihl RO, Benkelfat C (1994). Serotonin and alcohol intake, abuse, and dependence: clinical evidence. Biol Psychiatry 36: 326-337.

Lewontin RC (1988). On measures of gametic disequilibrium. Genetics 120: 849-852.

Maffei C, Fossati A, Agostoni I, Barraco A, Bagnato M, Deborah D et al (1997). Interrater reliability and internal consistency of the Structured Clinical Interview for DSM-IV Axis II Personality Disorders (SCID-II), version 2.0. J Personality Disorders 11: 279-284.

Mestel R, Wäschenbach T, Lutze C (2001). Interraterreliabilität für das SKID-II für Persönlichkeitsstörungen nach DSM-IV. In: DGPs (Hrsg). Psychologie 2000. Pabst Science Publishers: Lengerich.

Murphy DL (1990). Neuropsychiatric disorders and the multiple human brain serotonin receptor subtypes and subsystems. Neuropsychopharmacology 3: 457-471.

Murphy GE, Wetzel RD (1990). The lifetime risk of suicide in alcoholism. Arch Gen Psychiatry 47: 383-392.

Naranjo CA, Sellers EM, Lawrin MO (1986). Modulation of ethanol intake by serotonin uptake inhibitors. J Clin Psychiatry 47(Suppl): 16-22.

Nielsen DA, Virkkunen M, Lappalainen J, Eggert M, Brown GL, Long JC et al (1998). A tryptophan hydroxylase gene marker for suicidality and alcoholism. Arch Gen Psychiatry 55: 593-602.

Parsian A, Cloninger CR (2001). Serotonergic pathway genes and subtypes of alcoholism: association studies. Psychiatr Genet 11: 89-94.

Preuss UW, Schuckit MA, Smith TL, Danko GP, Buckman K, Bierut L et al (2002). Comparison of 3190 alcohol-dependent individuals with and without suicide attempts. Alcohol Clin Exp Res 26: 471-477.

Purcell S, Cherny SS, Sham PC (2003). Genetic Power Calculator: design of linkage and association genetic mapping studies of complex traits. Bioinformatics 19: 149-150.
Rice JP, Reich T, Bucholz KK, Neuman RJ, Fishman R, Rochberg N et al (1995). Comparison of direct interview and family history diagnoses of alcohol dependence. Alcohol Clin Exp Res 19: 1018-1023.

Reich DE, Cargill M, Bolk S, Ireland J, Sabeti PC, Richter DJ et al (2001). Linkage disequilibrium in the human genome. Nature 411: 199-204.

Schulze TG, McMahon FJ (2002). Genetic association mapping at the crossroads: which test and why? Overview and practical guidelines. Am J Med Genet 114: 1-11.

Sham PC, Curtis D (1995). Monte Carlo tests for associations between disease and alleles at highly polymorphic loci. Ann Hum Genet 59(Part 1): 97-105.

Stephens M, Smith NJ, Donnelly P (2001). A new statistical method for haplotype reconstruction from population data. Am J Hum Genet 68: 978-989.

Thompson EA, Deeb S, Walker D, Motulsky AG (1988). The detection of linkage disequilibrium between closely linked markers: RFLPs at the AI-CIII apolipoprotein genes. $A m \mathrm{~J}$ Hum Genet 42: 113-124.

Turner D, Choudhury F, Reynard M, Railton D, Navarrete C (2002). Typing of multiple single nucleotide polymorphisms in cytokine and receptor genes using SNaPshot. Hum Immunol 63: 508-513.

Virkkunen M, Linnoila M (1997). Serotonin in early-onset alcoholism. Recent Dev Alcohol 13: 173-189.

Walther DJ, Peter JU, Bashammakh S, Hortnagl H, Voits M, Fink H et al (2003). Synthesis of serotonin by a second tryptophan hydroxylase isoform. Science 299: 76.

Wittchen HU, Zaudig M, Fydrich T (1997). SKID-I/II: Strukturiertes klinisches Interview für DSM-IV. Hogrefe: Göttingen.

Zanarini MC, Skodol AE, Bender D, Dolan R, Sanislow C, Schaefer $\mathrm{E}$ et al (2000). The Collaborative Longitudinal Personality Disorders Study: reliability of axis I and II diagnoses. J Personality Disorders 14: 291-299.

Zhang X, Gainetdinov RR, Beaulieu JM, Sotnikova TD, Burch LH, Williams RB et al (2005). Loss-of-function mutation in tryptophan hydroxylase-2 identified in unipolar major depression. Neuron 45: 11-16.

Zhou Z, Roy A, Lipsky R, Kuchipudi K, Zhu G, Taubman J et al (2005). Haplotype-based linkage of tryptophan hydroxylase 2 to suicide attempt, major depression, and cerebrospinal fluid 5-hydroxyindoleacetic acid in 4 populations. Arch Gen Psychiatry 62: 1109-1118.

Zill P, Baghai TC, Zwanzger P, Schule C, Eser D, Rupprecht R et al (2004a). SNP and haplotype analysis of a novel tryptophan hydroxylase isoform (TPH2) gene provide evidence for association with major depression. Mol Psychiatry 9: 1030-1036.

Zill P, Buttner A, Eisenmenger W, Bondy B, Ackenheil M (2004b). Regional mRNA expression of a second tryptophan hydroxylase isoform in postmortem tissue samples of two human brains. Eur Neuropsychopharmacol 14: 282-284.

Zill P, Buttner A, Eisenmenger W, Moller HJ, Ackenheil M, Bondy B (2007). Analysis of tryptophan hydroxylase I and II mRNA expression in the human brain: A post-mortem study. J Psychiatr Res 41: 168-173.

Zill P, Buttner A, Eisenmenger W, Moller HJ, Bondy B, Ackenheil M (2004c). Single nucleotide polymorphism and haplotype analysis of a novel tryptophan hydroxylase isoform (TPH2) gene in suicide victims. Biol Psychiatry 56: 581-586. 